\title{
INFLUENCE OF BROTH ENRICHMENT AS WELL AS STORAGE AND TRANSPORT TIME ON THE SENSITIVITY OF MRSA SURVEILLANCE IN THE TROPICS
}

\author{
Hagen Frickmann ${ }^{1,2, *}$, Andreas Hahn ${ }^{3}$, Norbert Georg Schwarz ${ }^{4}$, Ralf Matthias Hagen ${ }^{1,5}$, Denise Dekker ${ }^{4}$, \\ Rebecca Hinz ${ }^{6}$, Volker Micheel ${ }^{7}$, Benedikt Hogan ${ }^{4}$, Jürgen May $^{4}$, Raphael Rakotozandrindrainy ${ }^{8}$ \\ ${ }^{1}$ Department of Tropical Medicine at the Bernhard Nocht Institute, Bundeswehr Hospital Hamburg, Hamburg, Germany \\ ${ }^{2}$ Institute for Medical Microbiology, Virology and Hygiene, University Medicine Rostock, Rostock, Germany \\ ${ }^{3}$ Institute for Microbiology, Charité - University Medicine Berlin, Berlin, Germany \\ ${ }^{4}$ Infectious Disease Epidemiology, Bernhard Nocht Institute for Tropical Medicine Hamburg, Hamburg, Germany \\ ${ }^{5}$ Department of Preventive Medicine, Bundeswehr Medical Academy, Munich, Germany \\ ${ }^{6}$ Institute for Medical Microbiology, Virology and Hygiene, University Medical Center Hamburg-Eppendorf, Hamburg, Germany \\ ${ }^{7}$ Central Institute of the Bundeswehr Medical Service Koblenz, Koblenz, Germany \\ ${ }^{8}$ Department of Microbiology and Parasitology, University of Antananarivo, Antananarivo, Madagascar
}

Received: August 28, 2017; Accepted: September 8, 2017

Direct growth on blood and screening agar for methicillin-resistant Staphylococcus aureus (MRSA) at a tropical surveillance site was compared with broth enrichment and subsequent growth on selective MRSA agar after international sample transport.

In Madagascar, 1548 swabs from an MRSA surveillance study were assessed for growth on Columbia blood agar enriched with 5\% sheep blood and MRSA screening agar at the surveillance site with subsequent cold storage of the samples and shipment to Germany. In Germany, 1541 shipped samples were analyzed by non-selective broth enrichment with subsequent culture on MRSA selective agar.

A total of 28 MRSA isolates were detected. Of these, 20 strains were isolated from direct culture on blood and MRSA screening agars at the surveillance site, 24 MRSA strains were isolated using the broth enrichment method in Germany, and 16 MRSA strains were identified by both approaches.

In spite of the observed die-off of individual strains due to long-term storage and transport, broth enrichment with subsequent screening on MRSA selective agar after international sample shipment led to comparable sensitivity of MRSA detection like streaking on blood and MRSA agar at the tropical surveillance site.

Keywords: MRSA screening, tropics, broth enrichment, storage, transport, pre-analytics

\section{Introduction}

Methicillin-resistant Staphylococcus aureus (MRSA) has been identified as a hygiene problem of importance in tropical settings $[1,2]$. The significance of respective surveillance has been emphasized since the last 15 years [1]. However, from many resource-limited tropical settings, reliable surveillance data on colonization and infection with MRSA are still scarce. Currently, available data derive mainly from cross-sectional studies and thus represent no more than a momentary status [3].

When assessing MRSA colonization, screening is typically based on swabbing of frequently colonized sites like the anterior nostrils. However, as recently demonstrated, the reliability of swabbing largely depends both on the correctly applied swabbing technique and the type

\footnotetext{
* Corresponding author: Hagen Frickmann; Department of Tropical Medicine at the Bernhard Nocht Institute, German Armed Forces Hospital of Hamburg, Bernhard Nocht Str. 74, 20359 Hamburg, Germany; Phone: 0049-40-6947-28700; Fax: 0049-40-6947-28709; Email: Frickmann@bni-hamburg.de

This is an open-access article distributed under the terms of the Creative Commons Attribution-NonCommercial 4.0 International License (https://creativecommons.org/licenses/by-nc/4.0/), which permits unrestricted use, distribution, and reproduction in any medium for non-commercial purposes, provided the original author and source are credited, a link to the CC License is provided, and changes - if any - are indicated.
} 
of swab used [4-7]. The heterogeneity of applied procedures makes a comparison between studies difficult and thus further limits the reliability of available data from the literature.

As shown in previous studies, the sensitivity of MRSA screening may be increased by broth enrichment [8-10]. However, broth enrichment requires considerable logistic efforts, including preparation, storage, and transport, that are difficult to guarantee in resource-limited tropical settings while ready-to-use agar preparations can comparably easily be provided [11].

If broth enrichment at the surveillance site in the tropics is not feasible for logistic reasons, sample transport to a well-equipped laboratory with subsequent broth enrichment may be performed. However, in case of prolonged sample storage and transport time, reduced sensitivity due to the die-off of bacterial cells has to be considered [12].

In order to assess potential beneficial and unfavorable effects of international sample transport with subsequent broth enrichment during surveillance studies in the tropical setting, direct growth on non-selective and selective agar was compared with sample transport by international flight with subsequent broth enrichment and culture on MRSA screening agar with sample material from the same study. This comparison also included an assessment of spa types to identify potential spa type-specific differences in storage and transport stability of MRSA strains as well as an assessment of the effect of the duration of storage and transport time.

\section{Materials and methods}

\section{Origin of specimens and applied screening procedures}

In a recently published study from Madagascar, 1548 nasal swabs (Copan Nasal Swab Transsystem ${ }^{\circledR}$, Brescia, Italy) were collected from students and health care workers [11]. Plating on Columbia blood agar (OXOID ${ }^{\circledR}$, Vienna, Austria) with subsequent biochemical identification and antibiotic resistance testing to identify MRSA isolates was performed as described by Hogan et al. [11]. For this integrated study, MRSA selective agar (CHROMagar MRSA, MAST Diagnostica, Reinfeld, Germany) was used on site in addition to the methods as described by Hogan et al. Afterwards, the same swabs were stored at $4{ }^{\circ} \mathrm{C}$ for $2-22$ weeks until airborne shipment without cooling for second processing to Germany.

A total of 1541 nasal swabs were shipped to the Bernhard Nocht Institute for Tropical Medicine Hamburg, Germany, and were analyzed by non-selective broth enrichment in thioglycolate broth (BD, Heidelberg, Germany) and subsequent culture on MRSA selective agar CHROMagar MRSA (MAST Diagnostica). The remaining 7 swabs were lost, so the samples could not be included in the assessment in Germany. MRSA strains were identified by matrix-assisted laser desorption-ionization time-offlight mass spectrometry (MALDI-TOF MS), by antibiotic susceptibility testing and by polymerase chain reaction (PCR) as detailed elsewhere [13].

spa typing

MRSA isolates that were identified by any of the approaches were assessed by spa typing as described elsewhere [11, 13] to assess potential clonal differences between strains identified by the different approaches.

\section{Impact of storage and transport time}

To assess the impact of storage and transport time on the reliability of MRSA recovery by broth enrichment after sample transport, three groups were compared taking the number of days between swab acquisition and broth enrichment in Germany into account: MRSA growth in Madagascar and after broth enrichment in Germany (group 1), MRSA growth in Madagascar only (group 2), and MRSA growth after broth enrichment in Germany only (group 3).

\section{Statistics}

Confidence intervals of the sensitivity and positivity rates of the different approaches of bacterial culture in Madagascar and in Germany were calculated with the software SAS version 9.4 (SAS Institute Inc., Cary, USA). The total number of MRSA-positive samples as identified by the application of both screening procedures was used as denominator for the calculation of sensitivity and positivity rate.

A Kruskal-Wallis test (GraphPad InStat ${ }^{\circledR}$, version 3.06, 32 bit for Windows, GraphPad Software, Inc., San Diego, USA) was performed to evaluate the impact of storage and transport time on the recovery of MRSA isolates from the swabs after transfer to Germany.

\section{Ethics statement}

Ethical clearance for the study was obtained from the Ethical Committee of the Ministry of Health of the Republic of Madagascar (no. 081 - MSANP/CE, 5 November 2012).

\section{Results}

\section{Numbers of MRSA strains identified in Madagascar and in Germany}

The combined selective and non-selective agar-based approach in Madagascar led to the identification of 20 (1.3\%) MRSA isolates in the 1541 nasal swabs. Broth enrichment with subsequent growth on selective agar led to the identification of 24 MRSA isolates (1.6\%). Both procedures led to matching positive results in $16(57.1 \%)$ out of 28 
Table 1. Detection of MRSA strains without broth enrichment at the surveillance site and with broth enrichment after international sample transport. A total of 1541 out of 1548 samples could be included in the assessment

\begin{tabular}{lccc}
\hline & MRSA with enrichment & No MRSA with enrichment & Total \\
\hline MRSA without enrichment & $16(1.0 \%)$ & $4(0.3 \%)$ & $20(1.3 \%)$ \\
No MRSA without enrichment & $8(0.5 \%)$ & $1,513(98.2 \%)$ & $1,521(98.7 \%)$ \\
Total & $24(1.6 \%)$ & $1,517(98.4 \%)$ & $1,541(100 \%)$ \\
\hline
\end{tabular}

instances of MRSA detection. In case of 4 swabs (14.3\%), only the agar-based approach in Madagascar led to the identification of MRSA. In 8 instances $(28.6 \%)$, only the enrichment procedure in Germany allowed the detection of MRSA strains. In total, 28 (1.8\%) out of 1541 swabs showed positive results for MRSA (Table 1).

Accordingly, sensitivity without broth enrichment in Madagascar was 0.71 (95\% confidence interval [CI], $0.51-0.87)$, sensitivity with broth enrichment in Germany was 0.86 (95\% CI, 0.67-0.96). Positivity rates were 0.013 for Madagascar (95\% CI, 0.008-0.020) and 0.016 for Germany (95\% CI, $0.010-0.023) . \kappa$ of 0.003 (95\% CI, -0.006 to 0.011 ) for matching of both tests was non-interpretable due to the low positivity rates.

spa type distribution of the additionally identified strains by the different approaches

The spa type distribution of the additionally identified MRSA strains either in Madagascar or in Germany did not show individual spa types with increased or decreased transport and storage stability but give an overview of circulating spa types in this region of Madagascar. In both instances, the frequently observed spa type t168 represented 3 quarters of the newly identified strains while the remaining quarter consisted of individual rare spa types (Table 2).

\section{Impact of storage and transport time}

Time until transport to Germany and further processing varied between 11 and 149 days. In detail, the mean time spans between sample acquisition in Madagascar and broth culture in Germany (in days \pm standard deviation [SD]) were $99 \pm 42$ days for strains that grew both in Madagascar and after broth enrichment in Germany, $133 \pm 17$ days for strains that grew in Madagascar only, and $110 \pm 36$ days for strains that grew after broth enrichment in Germany only. Kruskal-Wallis testing showed no significant differ- ence between the groups $(P=0.27, \mathrm{KW}=2.655$ [corrected for ties])

\section{Discussion}

The methodological comparison demonstrates that the broth enrichment approach leads to a comparable sensitivity during MRSA surveillance studies, even with considerable storage and transport time of specimens. These results are surprising, because long storage prior to international sample shipment might have led to a more pronounced dieoff of $S$. aureus. Previous studies suggested long-term effects [12]. Although the phenomenon of die-off of isolates was indeed observed, as 4 out of $28 \mathrm{~S}$. aureus were not detectable after transport to Germany by broth enrichment, its quantitative importance seems to be low. Of course, the low number of detected positive samples makes any definite conclusions challenging.

With focus on the isolates' spa types, no differing conclusions can be drawn. The only spa type of quantitative importance was t186. Virtually the same or a slightly higher sensitivity after broth enrichment in Germany was observed, reflecting the generally observed phenomenon.

Interestingly, there was no significant difference of the time spans between sample acquisition and onset of broth enrichment for MRSA strains that grew both in Madagascar and after broth enrichment in Germany, that grew in Madagascar only, and that grew in Germany after broth enrichment only. This result is surprising, because it challenges previous assessments that suggested quantitatively relevant effects on the die-off of staphylococci in case of prolonged storage and transport time [12]. A potential minor tendency of longer time spans in case of strains that did not grow any more after transport to Germany could not be statistically confirmed due to the low total number of isolates.

Previous studies [8-10] suggest that beneficial effects of broth enrichment regarding the diagnostic sensitivity are pronounced when die-off due to long storage and transport time can be avoided and broth culture can be performed di-

Table 2. spa type distribution of the identified MRSA strains. No hints for increased or decreased transport and storage stability of individual spa types are detectable

\begin{tabular}{lll}
\hline $\begin{array}{l}\text { spa types of strains that were detected } \\
\text { in Madagascar and in Germany }\end{array}$ & $\begin{array}{l}\text { spa types of strains that were detected } \\
\text { in Madagascar only }\end{array}$ & $\begin{array}{l}\text { spa types of strains that were detected } \\
\text { in Germany only }\end{array}$ \\
\hline $\begin{array}{l}\mathrm{t} 168(n=13), \mathrm{t} 2393(n=1), \mathrm{t} 5772 \\
(n=1), \mathrm{t} 13653(n=1)\end{array}$ & $\mathrm{t} 168(n=3), \mathrm{t} 5562(n=1)$ & $\mathrm{t} 168(n=6), \mathrm{t} 5772(n=1), \mathrm{t} 11285(n=1)$ \\
\hline
\end{tabular}


rectly at the study site. The here presented data set does not allow any conclusions regarding this issue. As an undeniable limitation of the study, the number of strains that were missed by both compared approaches remains unclear. The low number of positive samples is another limitation of the study which weakens any resulting recommendations.

Of note, PCR-based screenings for MRSA have been described as reliable and both cost- and time-efficient $[14,15]$, although these aspects may depend on the local prevalence and are therefore not yet generally accepted. However, such procedures might be a useful alternative if storage is required and die-off of isolates as to be considered. Taking the costs of such molecular approaches into account, their application in resource-limited tropical settings seems hardly realistic so far.

\section{Conclusions}

As a consequence of the results, a combined approach of direct agar culture and broth enrichment seems advisable. If possible for logistic reasons, broth enrichment should be performed directly at the study site.

\section{Funding sources}

There was no source of funding.

\section{Conflict of interest}

The authors declare that there are no conflicts of interest.

\section{References}

1. Shears P: Antibiotic resistance in the tropics. Epidemiology and surveillance of antimicrobial resistance in the tropics. Trans R Soc Trop Med Hyg 95,127-130 (2001)

2. Tong SY, Steer AC, Jenney AW, Carapetis JR: Communityassociated methicillin-resistant Staphylococcus aureus skin infections in the tropics. Dermatol Clin 29, 21-32 (2011)

3. Breurec S, Zriouil SB, Fall C, Boisier P, Brisse S, Djibo S, Etienne J, Fonkoua MC, Perrier-Gros-Claude JD, Pouillot R, Ramarokoto CE, Randrianirina F, Tall A, Thiberge JM, Working Group on Staphylococcus aureus infections, Laurent F, Garin B: Epidemiology of methicillin-resistant Staphylococcus aureus lineages in five major African towns: emergence and spread of atypical clones. Clin Microbiol Infect 17, 160-165 (2011)

4. Warnke P, Devide A, Weise M, Frickmann H, Schwarz NG, Schäffler H, Ottl P, Podbielski A: Utilizing moist or dry swabs for the sampling of nasal MRSA carriers? An in vivo and in vitro study. PLoS One 11, e0163073 (2016)

5. Warnke P, Frickmann H, Ottl P, Podbielski A: Nasal screening for MRSA: different swabs - different results! PLoS One 9, e111627 (2014)

6. Warnke P, Harnack T, Ottl P, Kundt G, Podbielski A: Nasal screening for Staphylococcus aureus - daily routine with improvement potentials. PLoS One 9, e89667 (2014)

7. Warnke P, Warning L, Podbielski A: Some are more equal - a comparative study on swab uptake and release of bacterial suspensions. PLoS One 9, e102215 (2014)

8. Wertheim H, Verbrugh HA, van Pelt C, de Man P, van Belkum A, Vos MC: Improved detection of methicillin-resistant Staphylococcus aureus using phenyl mannitol broth containing aztreonam and ceftizoxime. J Clin Microbiol 39, 2660-2662 (2001)

9. Fang H, Hedin G: Use of cefoxitin-based selective broth for improved detection of methicillin-resistant Staphylococcus aureus. J Clin Microbiol 44, 592-594 (2006)

10. French GL: Methods for screening for methicillin-resistant Staphylococcus aureus carriage. Clin Microbiol Infect 15 Suppl 7, 10-16 (2009)

11. Hogan B, Rakotozandrindrainy R, Al-Emran H, Dekker D, Hahn A, Jaeger A, Poppert S, Frickmann H, Hagen RM, Micheel V, Crusius S, Heriniaina JN, Rakotondrainiarivelo JP, Razafindrabe T, May J, Schwarz NG: Prevalence of nasal colonisation by methicillin-sensitive and methicillinresistant Staphylococcus aureus among healthcare workers and students in Madagascar. BMC Infect Dis 16, 420 (2016)

12. Tan TY, Ng LS, Sim DM, Cheng Y, Min MO: Evaluation of bacterial recovery and viability from three different swab transport systems. Pathology 46, 230-233 (2014)

13. Micheel V, Hogan B, Köller T, Warnke P, Crusius S, Hinz R, Hagen RM, Schwarz NG, Frickmann H: Screening agars for MRSA: evaluation of a stepwise diagnostic approach with two different selective agars for the screening for methicillin-resistant Staphylococcus aureus (MRSA). Mil Med Res 2, 18 (2015)

14. Shenoy ES, Kim J, Rosenberg ES, Cotter JA, Lee H, Walensky RP, Hooper DC: Discontinuation of contact precautions for methicillin-resistant Staphylococcus aureus: a randomized controlled trial comparing passive and active screening with culture and polymerase chain reaction. Clin Infect Dis 57, 176-184 (2013)

15. Shenoy ES, Lee H, Cotter JA, Ware W, Kelbaugh D, Weil E, Walensky RP, Hooper DC: Impact of rapid screening for discontinuation of methicillin-resistant Staphylococcus aureus contact precautions. Am J Infect Control 44, 215221 (2016) 